\title{
Capsule Commentary on Zhang et al., Combined healthy lifestyle behaviors and disability-free survival: the Ohsaki Cohort 2006 Study
}

\author{
Anuoluwapo Adelodun, MD MPH \\ University of Texas Southwestern Medical Center, Dallas, TX, USA.
}

$\mathrm{J}$ Gen Intern Med 34(9):1850

DOI: $10.1007 / \mathrm{s} 11606-019-05144-\mathrm{x}$

(c) Society of General Internal Medicine 2019

$\mathrm{T}$ his prospective cohort study by Zhang et al. ${ }^{1}$ examined the impact of healthy lifestyle (HL) behaviors on disability-free survival among elderly patients (aged > 65 years) in Oshaki City, Northeast Japan. A survey examining multiple lifestyle behaviors like smoking, exercise, fruit intake, and sleep habits was obtained at baseline during a twoweek window after which participants were followed up for 10 years. The result was an increase in disability-free survival by 17.1 months in those who adhered to $3 \mathrm{HL}$ behaviors compared with those who adhered to only 0 or 1 behaviors. The study used the LTCI, a mandatory form of national social insurance in Japan. Age or the presence of chronic conditions did not seem to play a role in the effectiveness of HL behaviors as both late elderly ( $\geq 75$ years) and elderly patients having chronic conditions showed similar benefits from HL behaviors.

However, documentation of HL behaviors was only obtained at baseline but not during the 10-year follow-up; thus, it is unclear if these behaviors were consistent throughout the study period. Previous studies have alluded to the fact that $\mathrm{HL}$ behaviors were associated with increased survival ${ }^{2}$ and a reduction in all-cause mortality risk ${ }^{3}$ but none had been done on disability-free survival. ${ }^{1}$ To move the field forward, new researchers should carry out similar prospective cohort studies where HL behaviors are measured at baseline and monitored throughout the follow-up period. In addition, new HL behaviors should be examined to determine how they affect survival and mortality risk.
For clinicians, this study provides a strong reference for patient education on the benefit of a healthy lifestyle in the elderly. Other populations should be studied and additional HL behaviors like a low salt $\operatorname{diet}^{4}$ and reduced alcohol consumption ${ }^{5}$ explored.

Corresponding Author: Anuoluwapo Adelodun, MD MPH; University of Texas Southwestern Medical Center, Dallas, TX, USA (e-mail: anuoluwapo.adelodun@utsouthwestern.edu).

\section{Compliance with Ethical Standards:}

Conflict of Interest: The author declares that she does not have a conflict of interest.

\section{REFERENCES}

1. Zhang S, Tomata Y, Discacciati A, Otsuka T, Sugawara Y, Tanji F, Tsuji I. Combined healthy lifestyle behaviors and disability-free survival: the Ohsaki Cohort 2006 Study. J Gen Intern Med. DOI: https://doi.org/10. 1007/s11606-019-05061-Z

2. Rizzuto D, Orsini N, Giu C, Wang H-X, Fratiglioni L. Lifestyle, social factors, and survival after age 75: population based study. BMJ. 2012;345. doi:https://doi.org/10.1136/bmj.e5568

3. Loef $\mathbf{M}$, Walach $\mathbf{H}$. The combined effects of healthy lifestyle behaviors on all-cause mortality: a systematic review and meta-analysis. Preventive medicine. 2012;55(3): 163-70.

4. Larsson SC, Kaluza J, Wolk A. Combined impact of healthy lifestyle factors on lifespan: two prospective cohorts. Journal of internal medicine. 2017;282(3):209-19.

5. Tsubono, Y., Koizumi, Y., Nakaya, N., et al.. Health practices and mortality in Japan: combined effects of smoking, drinking, walking and body mass index in the Miyagi Cohort Study. J. Epidemiol. 2004; 14 (Suppl. 1), S39-S45.

Publisher's Note Springer Nature remains neutral with regard to jurisdictional claims in published maps and institutional affiliations. 